ANNALES

POLONICI MATHEMATICI

$98.3(2010)$

\title{
Exponential convergence of shunting inhibitory cellular neural networks with continuously distributed delays
}

\author{
by Qiyuan Zhou and Changhong Zhao (Changde)
}

\begin{abstract}
We study delay shunting inhibitory cellular neural networks without almost periodic coefficients. Some sufficient conditions are established to ensure that all solutions of the networks converge exponentially to an almost periodic function. This complements previously known results.
\end{abstract}

1. Introduction. Consider the shunting inhibitory cellular neural networks (SICNNs) with continuously distributed delays given by

$$
\begin{aligned}
x_{i j}^{\prime}(t)= & -a_{i j}(t) x_{i j}(t)-\sum_{C_{k l} \in N_{r}(i, j)} C_{i j}^{k l}(t) \int_{0}^{\infty} K_{i j}(u) f\left(x_{k l}(t-u)\right) d u x_{i j}(t) \\
& +L_{i j}(t), \quad i=1, \ldots, m, j=1, \ldots, n,
\end{aligned}
$$

where $C_{i j}$ denotes the cell at the $(i, j)$ position of the lattice, the $r$-neighborhood $N_{r}(i, j)$ of $C_{i j}$ is

$$
N_{r}(i, j)=\left\{C_{k l}: \max (|k-i|,|l-j|) \leq r, 1 \leq k \leq m, 1 \leq l \leq n\right\},
$$

$x_{i j}$ is the activity of the cell $C_{i j}, L_{i j}(t)$ is the external input to $C_{i j}, a_{i j}(t)>0$ represents the passive decay rate of the cell activity at time $t, C_{i j}^{k l}(t) \geq 0$ is the connection or coupling strength of postsynaptic activity of the cell transmitted to the cell $C_{i j}$ at time $t$, and the activity function $f(\cdot)$ is a continuous function representing the output or firing rate of the cell $C_{k l}$.

Since Bouzerdoum and Pinter [1-3] described SICNNs as new cellular neural networks (CNNs), SICNNs have been extensively applied in psychophysics, speech, perception, robotics, adaptive pattern recognition, vision, and image processing. Hence, they have been the object of intensive analysis by numerous authors in recent years. In particular, there have been extensive results on the problem of the existence and stability of almost pe-

2010 Mathematics Subject Classification: 34C25, 34K13, 34K25.

Key words and phrases: shunting inhibitory cellular neural networks, exponential convergence, continuously distributed delays. 
riodic solutions for system (1.1) in the literature. We refer the reader to [4, $5,9,10,11,13]$ and the references cited therein. Suppose that the following condition is satisfied:

$\left(H_{0}\right) a_{i j}, C_{i j}^{k l}, L_{i j}: \mathbb{R} \rightarrow \mathbb{R}$ are almost periodic functions, where $i=$ $1, \ldots, m, j=1, \ldots, n$.

Most authors cited above then deduced that all solutions of system (1.1) converge exponentially to an almost periodic function. However, to the best of our knowledge, few authors have considered the convergence behavior for all solutions of system (1.1) without the assumption $\left(H_{0}\right)$. Thus, it is worthwhile to investigate the convergence behavior for all solutions of system (1.1) in this case.

The main purpose of this paper is to study system (1.1) with almost periodic coefficients being perturbed by exponentially small perturbations as $t \rightarrow+\infty$. By applying techniques similar to those in [12], we derive some sufficient conditions ensuring that all solutions of system (1.1) converge exponentially to an almost periodic function. Moreover, an example is provided to illustrate the effectiveness of our results.

Consider the following delayed shunting inhibitory cellular neural networks:

$$
\begin{aligned}
x_{i j}^{\prime}(t)= & -a_{i j}^{*}(t) x_{i j}(t)-\sum_{C_{k l} \in N_{r}(i, j)} \tilde{C}_{i j}^{k l}(t) \int_{0}^{\infty} K_{i j}(u) f\left(x_{k l}(t-u)\right) d u x_{i j}(t) \\
& +L_{i j}^{*}(t), \quad i=1, \ldots, m, j=1, \ldots, n .
\end{aligned}
$$

Throughout this paper, for $i=1, \ldots, m, j=1, \ldots, n$, it will be assumed that $a_{i j}^{*}, \tilde{C}_{i j}^{k l}, L_{i j}^{*}: \mathbb{R} \rightarrow \mathbb{R}$ are almost periodic functions such that

$$
\left\{\begin{array}{l}
0<\underline{a}_{i j}:=\inf _{t \in \mathbb{R}} a_{i j}^{*}(t)<+\infty, \quad \bar{C}_{i j}^{k l}:=\sup _{t \in \mathbb{R}} \tilde{C}_{i j}^{k l}(t)<+\infty, \\
L_{i j}^{+}:=\sup _{t \in \mathbb{R}}\left|L_{i j}^{*}(t)\right|<+\infty .
\end{array}\right.
$$

Throughout, we set

$$
\left\{x_{i j}(t)\right\}=\left(x_{11}(t), \ldots, x_{1 n}(t), \ldots, x_{i 1}(t), \ldots, x_{i n}(t), \ldots, x_{m 1}(t), \ldots, x_{m n}(t)\right) .
$$

For $x(t)=\left\{x_{i j}(t)\right\} \in \mathbb{R}^{m \times n}$, we define the norm $\|x(t)\|=\max _{(i, j)}\left\{\left|x_{i j}(t)\right|\right\}$. If $g_{1}, g_{2}: \mathbb{R} \rightarrow \mathbb{R}$ are continuous, and there exist constants $\zeta, \delta>0$ such that

$$
\left|g_{1}(t) / g_{2}(t)\right| \leq \zeta \quad \text { for all } t \geq \delta,
$$

we write $g_{1}(t)=O\left(g_{2}(t)\right)$.

We also assume that the following conditions hold:

$\left(H_{1}\right)$ For $i \in\{1, \ldots, m\}, j \in\{1, \ldots, n\}$, the delay kernels $K_{i j}:[0, \infty) \rightarrow$ $\mathbb{R}$ are continuous, integrable and there exist constants $k_{i j} \geq 0$ and 
$\lambda>0$ such that

$$
\int_{0}^{\infty}\left|K_{i j}(s)\right| d s \leq k_{i j}, \quad \int_{0}^{\infty}\left|K_{i j}(u)\right| e^{\lambda u} d u<+\infty .
$$

$\left(H_{2}\right)$ There exist constants $M_{f}$ and $\mu_{f}$ such that

$$
|f(u)-f(v)| \leq \mu_{f}|u-v|, \quad|f(u)| \leq M_{f} \quad \text { for all } u, v \in \mathbb{R} .
$$

$\left(H_{3}\right)$ The quantities defined by

$$
L=\max _{(i, j)} \frac{L_{i j}^{+}}{\underline{a}_{i j}}, \quad \delta=\max _{(i, j)} \frac{\sum_{C_{k l} \in N_{r}(i, j)} \bar{C}_{i j}^{k l} M_{f} k_{i j}}{\underline{a}_{i j}}
$$

satisfy $0 \leq L<+\infty, 0<\delta<1$, and there exist constants $\eta, \lambda>0$ such that for all $i, j$ as above,

$$
\left(\lambda-\underline{a}_{i j}\right)+\sum_{C_{k l} \in N_{r}(i, j)} \bar{C}_{i j}^{k l}\left(M_{f} k_{i j}+\mu_{f} \int_{0}^{\infty}\left|K_{i j}(u)\right| e^{\lambda u} d u \frac{L}{1-\delta}\right)<-\eta<0 .
$$

$\left(H_{4}\right)$ For $i, j$ as above, $a_{i j}, C_{i j}^{k l}, L_{i j}: \mathbb{R} \rightarrow \mathbb{R}$ are continuous functions, and

$$
\begin{aligned}
a_{i j}(t)-a_{i j}^{*}(t) & =O\left(e^{-\lambda t}\right), L_{i j}(t)-L_{i j}^{*}(t)=O\left(e^{-\lambda t}\right), \\
C_{i j}^{k l}(t)-\tilde{C}_{i j}^{k l}(t) & =O\left(e^{-\lambda t}\right) .
\end{aligned}
$$

The initial conditions associated with system (1.1) are of the form

$$
x_{i j}(s)=\varphi_{i j}(s), \quad s \in(-\infty, 0], i=1, \ldots, m, j=1, \ldots, n,
$$

where $\varphi_{i j}(\cdot)$ denotes a real-valued bounded continuous function defined on $(-\infty, 0]$.

Definition 1 (see $[6,7]$ ). Let $u: \mathbb{R} \rightarrow \mathbb{R}^{m \times n}$ be continuous. Then $u$ is said to be almost periodic on $\mathbb{R}$ if, for any $\varepsilon>0$, the set $T(u, \varepsilon)=\{\delta$ : $\|u(t+\delta)-u(t)\|<\varepsilon, \forall t \in \mathbb{R}\}$ is relatively dense, i.e., for any $\varepsilon>0$, it is possible to find a real number $l=l(\varepsilon)>0$ such that for any interval with length $l(\varepsilon)$, there exists a number $\delta=\delta(\varepsilon)$ in this interval such that $\|u(t+\delta)-u(t)\|<\varepsilon$ for all $t \in \mathbb{R}$.

Since $a_{i j}^{*}, \tilde{C}_{i j}^{k l}$ and $L_{i j}^{*}$ are almost periodic functions, by using a similar argument to that in the proof of Theorem 2.1 in [13], we can easily show the following lemma.

Lemma 1.1. Let $\left(H_{1}\right)-\left(H_{3}\right)$ hold. Then system (1.2) has exactly one almost periodic solution $Z^{*}=\left\{x_{i j}^{*}(t)\right\}=\left(x_{11}^{*}(t), \ldots, x_{m n}^{*}(t)\right)$, and $\sup _{t \in \mathbb{R}}\left|x_{i j}^{*}(t)\right|$ $\leq L /(1-\delta), i=1, \ldots, m, j=1, \ldots, n$.

The remaining part of this paper is organized as follows. In Section 2, we present some new sufficient conditions to ensure that all solutions of system 
(1.1) converge exponentially to an almost periodic function. In Section 3, we give some examples and remarks to illustrate our results.

\section{Main results}

Theorem 2.1. Let $\left(H_{1}\right)-\left(H_{4}\right)$ hold. Suppose that $Z^{*}(t)=\left\{x_{i j}^{*}(t)\right\}=$ $\left(x_{11}^{*}(t), \ldots, x_{m n}^{*}(t)\right)$ is the almost periodic solution of system (1.2). Then, for every solution $Z(t)=\left\{x_{i j}(t)\right\}=\left(x_{11}(t), \ldots, x_{m n}(t)\right)$ of system (1.1) with any initial value $\varphi=\left\{\varphi_{i j}(t)\right\}=\left(\varphi_{11}(t), \ldots, \varphi_{m n}(t)\right)$, we have

$$
\left|x_{i j}(t)-x_{i j}^{*}(t)\right|=O\left(e^{-\lambda t}\right) \quad \text { for all } i, j .
$$

Proof. Set

$$
\begin{aligned}
\epsilon_{i j}(t)=- & {\left[a_{i j}(t)-a_{i j}^{*}(t)\right] x_{i j}^{*}(t)-\sum_{C_{k l} \in N_{r}(i, j)}\left[C_{i j}^{k l}(t)-\tilde{C}_{i j}^{k l}(t)\right] } \\
& \cdot \int_{0}^{\infty} K_{i j}(u) f\left(x_{k l}^{*}(t-u)\right) d u x_{i j}^{*}(t)+\left[L_{i j}(t)-L_{i j}^{*}(t)\right] .
\end{aligned}
$$

Since $Z^{*}(t)=\left(x_{11}^{*}(t), \ldots, x_{m n}^{*}(t)\right)$ is an almost periodic function, by $\left(H_{3}\right)$ and $\left(H_{4}\right)$, we can choose constants $F, T>0$ such that

$$
\left|\epsilon_{i j}(t)\right|<\frac{1}{2} F e^{-\lambda t} \quad \text { for all } t \geq T,
$$

and

$$
\left[\lambda-a_{i j}(t)\right]+\sum_{C_{k l} \in N_{r}(i, j)} C_{i j}^{k l}(t)\left[M_{f} k_{i j}+\mu_{f} \int_{0}^{\infty}\left|K_{i j}(u)\right| e^{\lambda u} d u \frac{L}{1-\delta}\right]
$$$$
<\left[\lambda-a_{i j}^{*}(t)\right]+\sum_{C_{k l} \in N_{r}(i, j)} \tilde{C}_{i j}^{k l}(t)\left[M_{f} k_{i j}+\mu_{f} \int_{0}^{\infty}\left|K_{i j}(u)\right| e^{\lambda u} d u \frac{L}{1-\delta}\right]+\frac{1}{2} \eta
$$$$
<\left[\lambda-\underline{a}_{i j}\right]+\sum_{C_{k l} \in N_{r}(i, j)} \bar{C}_{i j}^{k l}\left[M_{f} k_{i j}+\mu_{f} \int_{0}^{\infty}\left|K_{i j}(u)\right| e^{\lambda u} d u \frac{L}{1-\delta}\right]+\frac{1}{2} \eta
$$$$
<-\frac{1}{2} \eta
$$

for all $t \geq T$ and all $i, j$, where $T$ is a sufficiently large constant.

Let $Z(t)=\left\{x_{i j}(t)\right\}=\left(x_{11}(t), \ldots, x_{m n}(t)\right)$ be a solution of system (1.1) with any initial value $\varphi=\left\{\varphi_{i j}(t)\right\}=\left(\varphi_{11}(t), \ldots, \varphi_{m n}(t)\right)$, and define

$$
y(t)=\left\{y_{i j}(t)\right\}=\left(y_{11}(t), \ldots, y_{m n}(t)\right)=Z(t)-Z^{*}(t) .
$$


Then

(2.3) $\quad y_{i j}^{\prime}(t)$

$$
\begin{aligned}
= & -a_{i j}(t) y_{i j}(t)-\sum_{C_{k l} \in N_{r}(i, j)} C_{i j}^{k l}(t)\left[\int_{0}^{\infty} K_{i j}(u) f\left(x_{k l}(t-u)\right) d u x_{i j}(t)\right. \\
& \left.-\int_{0}^{\infty} K_{i j}(u) f\left(x_{k l}^{*}(t-u)\right) d u x_{i j}^{*}(t)\right]+\epsilon_{i j}(t)
\end{aligned}
$$

for all $i, j$. Let $(i j)_{t}$ be an index such that

$$
\left|y_{(i j)_{t}}(t)\right|=\|y(t)\| \text {. }
$$

Calculating the upper right derivative of $e^{\lambda s}\left|y_{(i j)_{s}}(s)\right|$, in view of $\left(H_{1}\right)$ and $\left(H_{2}\right)$, for all $t \geq T$, we have

$$
\begin{aligned}
& \left.D^{+}\left(e^{\lambda s}\left|y_{(i j)_{s}}(s)\right|\right)\right|_{s=t} \\
& =\lambda e^{\lambda t}\left|y_{(i j)_{t}}(t)\right|+e^{\lambda t} \operatorname{sign}\left(y_{(i j)_{t}}(t)\right)\left\{-a_{(i j)_{t}}(t) y_{(i j)_{t}}(t)\right. \\
& -\sum_{C_{k l} \in N_{r}(i, j)_{t}} C_{(i j)_{t}}^{k l}(t)\left[\int_{0}^{\infty} K_{(i j)_{t}}(u) f\left(x_{k l}(t-u)\right) d u x_{(i j)_{t}}(t)\right. \\
& \left.\left.-\int_{0}^{\infty} K_{(i j)_{t}}(u) f\left(x_{k l}^{*}(t-u)\right) d u x_{(i j)_{t}}^{*}(t)\right]+\epsilon_{(i j)_{t}}(t)\right\} \\
& \leq e^{\lambda t}\left\{\left[\lambda-a_{(i j)_{t}}(t)\right]\left|y_{(i j)_{t}}(t)\right|\right. \\
& +\sum_{C_{k l} \in N_{r}(i, j)_{t}} C_{(i j)_{t}}^{k l}(t)\left[\mid \int_{0}^{\infty} K_{(i j)_{t}}(u) f\left(x_{k l}(t-u)\right) d u x_{(i j)_{t}}(t)\right. \\
& -\int_{0}^{\infty} K_{(i j)_{t}}(u) f\left(x_{k l}(t-u)\right) d u x_{(i j)_{t}}^{*}(t) \\
& +\mid \int_{0}^{\infty} K_{(i j)_{t}}(u) f\left(x_{k l}(t-u)\right) d u x_{(i j)_{t}}^{*}(t) \\
& \left.\left.-\int_{0}^{\infty} K_{(i j)_{t}}(u) f\left(x_{k l}^{*}(t-u)\right) d u x_{(i j)_{t}}^{*}(t) \mid\right]+\left|\epsilon_{(i j)_{t}}(t)\right|\right\} \\
& <e^{\lambda t}\left\{\left[\lambda-a_{(i j)_{t}}(t)\right]\left|y_{(i j)_{t}}(t)\right|+\sum_{C_{k l} \in N_{r}(i, j)_{t}} C_{(i j)_{t}}^{k l}(t)\left[M_{f} k_{(i j)_{t}}\left|y_{(i j)_{t}}(t)\right|\right.\right. \\
& \left.\left.+\mu_{f} \int_{0}^{\infty}\left|K_{(i j)_{t}}(u)\right|\left|y_{k l}(t-u)\right| d u \frac{L}{1-\delta}\right]\right\}+\frac{1}{2} F \text {. }
\end{aligned}
$$


Let

$$
M(t)=\max _{s \leq t} e^{\lambda s}\|y(s)\|
$$

It is obvious that $e^{\lambda t}\|y(t)\| \leq M(t)$, and $M(t)$ is non-decreasing.

Now, we consider two cases.

CAse (i). Suppose that

$$
M(t)>e^{\lambda t}\|y(t)\| \quad \text { for all } t \geq T .
$$

Then we claim that

$$
M(t) \equiv M(T) \text { is a constant for all } t \geq T
$$

Assume, by way of contradiction, that (2.8) does not hold. Then there exists $t_{1}>T$ such that $M\left(t_{1}\right)>M(T)$. Since

$$
e^{\lambda t}\|y(t)\| \leq M(T) \quad \text { for all } t \leq T
$$

there must exist $\beta \in\left(T, t_{1}\right)$ such that

$$
e^{\lambda \beta}\|y(\beta)\|=M\left(t_{1}\right) \geq M(\beta),
$$

which contradicts (2.7). This contradiction implies that (2.8) holds. It follows that

$$
e^{\lambda t}\|y(t)\|<M(t)=M(T) \quad \text { for all } t \geq T \text {. }
$$

CASE (ii). Suppose that there is a point $t_{0} \geq T$ such that $M\left(t_{0}\right)=$ $e^{\lambda t_{0}}\left\|y\left(t_{0}\right)\right\|$. Then, in view of (2.2) and (2.5), we get

$$
\begin{aligned}
& <e^{\lambda t_{0}}\left\{\left[\lambda-a_{(i j)_{t_{0}}}\left(t_{0}\right)\right]\left|y_{(i j)_{t_{0}}}\left(t_{0}\right)\right|+\sum_{C_{k l} \in N_{r}(i, j)_{t_{0}}} C_{(i j)_{t_{0}}}^{k l}\left(t_{0}\right)\left[M_{f} k_{(i j)_{t_{0}}}\left|y_{(i j)_{t_{0}}}\left(t_{0}\right)\right|\right.\right. \\
& \left.\left.\quad+\mu_{f} \int_{0}^{\infty}\left|K_{(i j)_{t_{0}}}(u)\right|\left|y_{k l}\left(t_{0}-u\right)\right| d u \frac{L}{1-\delta}\right]\right\}+\frac{1}{2} F \\
& =\left[\lambda-a_{(i j)_{t_{0}}}\left(t_{0}\right)\right]\left|y_{(i j)_{t_{0}}}\left(t_{0}\right)\right| e^{\lambda t_{0}}+\sum_{C_{k l} \in N_{r}(i, j)_{t_{0}}} C_{(i j)_{t_{0}}}^{k l}(t)\left[M_{f} k_{(i j)_{t_{0}}}\left|y_{(i j)_{t_{0}}}\left(t_{0}\right)\right| e^{\lambda t_{0}}\right. \\
& \left.\quad+\mu_{f} \int_{0}^{\infty}\left|K_{(i j)_{t_{0}}}(u)\right| e^{\lambda u}\left|y_{k l}\left(t_{0}-u\right)\right| e^{\lambda\left(t_{0}-u\right)} d u \frac{L}{1-\delta}\right]+\frac{1}{2} F
\end{aligned}
$$




$$
\begin{aligned}
\leq & \left\{\left[\lambda-a_{(i j)_{t_{0}}}\left(t_{0}\right)\right]+\sum_{C_{k l} \in N_{r}(i, j)_{t_{0}}} C_{(i j)_{t_{0}}}^{k l}(t)\left[M_{f} k_{(i j)_{t_{0}}}\right.\right. \\
& \left.\left.+\mu_{f} \int_{0}^{\infty}\left|K_{(i j)_{t_{0}}}(u)\right| e^{\lambda u} d u \frac{L}{1-\delta}\right]\right\} M\left(t_{0}\right)+\frac{1}{2} F \\
\leq & -\frac{1}{2} \eta M\left(t_{0}\right)+\frac{1}{2} F .
\end{aligned}
$$

In addition, if $M\left(t_{0}\right) \geq F / \eta$, then $M(t)$ is strictly decreasing in a small neighborhood $\left(t_{0}, t_{0}+\delta_{0}\right)$. This contradicts that $M(t)$ is non-decreasing. Hence,

$$
e^{\lambda t_{0}}\left\|y\left(t_{0}\right)\right\|=M\left(t_{0}\right)<F / \eta .
$$

For all $t>t_{0}$, by the same approach used in the proof of (2.11), we have

$$
e^{\lambda t}\|y(t)\|<F / \eta \quad \text { if } M(t)=e^{\lambda t}\|y(t)\| .
$$

On the other hand, if $M(t)>e^{\lambda t}\|y(t)\|$ for $t>t_{0}$, we can choose $t_{0} \leq$ $t_{3}<t$ such that

$$
M\left(t_{3}\right)=e^{\lambda t_{3}}\left\|y\left(t_{3}\right)\right\|<F / \eta \quad \text { and } \quad M(s)>e^{\lambda s}\|y(s)\| \quad \text { for all } s \in\left(t_{3}, t\right] .
$$

Using a similar argument to the proof of Case (i), we can show that

$$
M(s) \equiv M\left(t_{3}\right) \text { is a constant for all } s \in\left(t_{3}, t\right],
$$

which implies that

$$
e^{\lambda t}\|y(t)\|<M(t)=M\left(t_{3}\right)<F / \eta .
$$

In summary, there must exist $N>0$ with $e^{\lambda t}\|y(t)\|<\max \{M(T), F / \eta\}$ for all $t>N$. This completes the proof of Theorem 2.1.

3. An example. In this section, we give an example to illustrate the results obtained in the previous sections.

ExAmple 3.1. Consider the following SICNNs with continuously distributed delays:

$$
\begin{array}{r}
\frac{d x_{i j}}{d t}=-a_{i j}(t) x_{i j}-\sum_{C_{k l} \in N_{r}(i, j)} C_{i j}^{k l}(t) \int_{0}^{\infty} K_{i j}(u) f\left(x_{k l}(t-u)\right) d u x_{i j}+L_{i j}(t), \\
i, j=1,2,3,
\end{array}
$$

where

$$
\left[\begin{array}{lll}
a_{11}(t) & a_{12}(t) & a_{13}(t) \\
a_{21}(t) & a_{22}(t) & a_{23}(t) \\
a_{31}(t) & a_{32}(t) & a_{33}(t)
\end{array}\right]=\left[\begin{array}{lll}
1+e^{-t} & 1+e^{-t} & 3+e^{-t} \\
3+e^{-t} & 1+e^{-t} & 3+e^{-t} \\
3+e^{-t} & 1+e^{-t} & 3+e^{-t}
\end{array}\right]
$$




$$
\left[\begin{array}{ccc}
c_{11} & c_{12} & c_{13} \\
c_{21} & c_{22} & c_{23} \\
c_{31} & c_{32} & c_{33}
\end{array}\right]=\left[\begin{array}{ccc}
0.1+e^{-2 t} & 0.2+e^{-2 t} & 0.1+e^{-2 t} \\
0.2+e^{-2 t} & 0+e^{-2 t} & 0.2+e^{-2 t} \\
0.1+e^{-2 t} & 0.2+e^{-2 t} & 0.1+e^{-2 t}
\end{array}\right]
$$

and

$$
\left[\begin{array}{lll}
L_{11} & L_{12} & L_{13} \\
L_{21} & L_{22} & L_{23} \\
L_{31} & L_{32} & L_{33}
\end{array}\right]
$$

$=\left[\begin{array}{ccc}0.7+0.24 \sin ^{2} \sqrt{2} t+e^{-2 t} & 0.41+0.5 \cos ^{2} t+e^{-2 t} & 0.94+2 \sin ^{2} t+e^{-2 t} \\ 0.91+2 \cos ^{2} t+e^{-2 t} & 0.67+0.2 \sin ^{2} t+e^{-2 t} & 0.91+2 \sin ^{2} t+e^{-2 t} \\ 2.54+0.4 \cos ^{4} t+e^{-2 t} & 0.5+0.41 \sin ^{2} t+e^{-2 t} & 0.94+2 \cos ^{2} t+e^{-2 t}\end{array}\right]$.

Let

$$
\begin{array}{r}
x_{i j}^{\prime}(t)=-a_{i j}^{*} x_{i j}(t)-\sum_{C_{k l} \in N_{r}(i, j)} \tilde{C}_{i j}^{k l} \int_{0}^{\infty} K_{i j}(u) f\left(x_{k l}(t-u)\right) d u x_{i j}(t)+L_{i j}^{*}(t), \\
i, j=1,2,3,
\end{array}
$$

where

$$
\begin{aligned}
& {\left[\begin{array}{lll}
a_{11}^{*} & a_{12}^{*} & a_{13}^{*} \\
a_{21}^{*} & a_{22}^{*} & a_{23}^{*} \\
a_{31}^{*} & a_{32}^{*} & a_{33}^{*}
\end{array}\right]=\left[\begin{array}{lll}
1 & 1 & 3 \\
3 & 1 & 3 \\
3 & 1 & 3
\end{array}\right]} \\
& {\left[\begin{array}{lll}
\tilde{c}_{11} & \tilde{c}_{12} & \tilde{c}_{13} \\
\tilde{c}_{21} & \tilde{c}_{22} & \tilde{c}_{23} \\
\tilde{c}_{31} & \tilde{c}_{32} & \tilde{c}_{33}
\end{array}\right]=\left[\begin{array}{ccc}
0.1 & 0.2 & 0.1 \\
0.2 & 0 & 0.2 \\
0.1 & 0.2 & 0.1
\end{array}\right],} \\
& {\left[\begin{array}{lll}
L_{11}^{*} & L_{12}^{*} & L_{13}^{*} \\
L_{21}^{*} & L_{22}^{*} & L_{23}^{*} \\
L_{31}^{*} & L_{32}^{*} & L_{33}^{*}
\end{array}\right]} \\
& =\left[\begin{array}{ccc}
0.7+0.24 \sin ^{2} \sqrt{2} t & 0.41+0.5 \cos ^{2} t & 0.94+2 \sin ^{2} t \\
0.91+2 \cos ^{2} t & 0.67+0.2 \sin ^{2} t & 0.91+2 \sin ^{2} t \\
2.54+0.4 \cos ^{4} t & 0.5+0.41 \sin ^{2} t & 0.94+2 \cos ^{2} t
\end{array}\right] .
\end{aligned}
$$

Set $r=1, K_{i j}(u)=e^{-2 u} \sin u, i, j=1,2,3$, and $f(x)=\frac{1}{20}(|x-1|-|x+1|)$. Then it is straightforward to check that the SICNNs (3.1) and (3.5) satisfy the conditions $\left(H_{1}\right)-\left(H_{4}\right)$. Hence, from Lemma 1.1 and Theorem 2.1, system 
(3.5) has exactly one almost periodic solution. Moreover, all solutions of system (3.1) converge exponentially to the almost periodic solution of system (3.5).

REMARK 3.1. System (3.1) is a very simple form of delayed shunting inhibitory neural networks without almost periodic coefficients. Therefore, the results in $[2-5,8-13]$ and the references therein cannot be applied to prove that all solutions of system (3.1) converge exponentially to an almost periodic function. This implies that the results of this paper are essentially new.

Acknowledgments. This work was supported by the Key Project of Chinese Ministry of Education (No. 2010151), the grants 06JJ2063, 07JJ6001 from the Scientific Research Fund of Hunan Provincial Natural Science Foundation, and 08C616, 09B072 from the Scientific Research Fund of Hunan Provincial Education Department of China.

\section{References}

[1] A. Bouzerdoum and R. B. Pinter, Shunting inhibitory cellular neural networks: derivation and stability analysis, IEEE Trans. Circuits Systems I Fund. Theory Appl. 40 (1993), 215-221.

[2] - - - Analysis and analog implementation of directionally sensitive shunting inhibitory Cellular Neural Networks, in: Visual Information Processing: From Neurons to Chips, SPIE 1473, 1991, 29-38.

[3] - - - Nonlinear lateral inhibition applied to motion detection in the fly visual system, in: Nonlinear Vision, R. B. Pinter and B. Nabet (eds.), CRC Press, Boca Raton, FL, 1992, 423-450.

[4] M. Cai and W. Xiong, Almost periodic solutions for shunting inhibitory cellular neural networks without global Lipschitz and bounded activation functions, Phys. Lett. A 362 (2007), 417-423.

[5] A. Chen, J. Cao and L. Huang, Almost periodic solution of shunting inhibitory CNNs with delays, ibid. 298 (2002), 161-170.

[6] A. M. Fink, Almost Periodic Differential Equations, Lecture Notes in Math. 377, Springer, Berlin, 1974.

[7] C. Y. He, Almost Periodic Differential Equations, Higher Education Publ. House, Beijing, 1992 (in Chinese).

[8] Y. Li, C. Liu and L. Zhu, Global exponential stability of periodic solution of shunting inhibitory CNNs with delays, Phys. Lett. A 337 (2005), 46-54.

[9] B. Liu and L. Huang, Existence and stability of almost periodic solutions for shunting inhibitory cellular neural networks with continuously distributed delays, ibid. 349 (2006), 177-186.

[10] Y. Liu, Z. You and L. Cao, On the almost periodic solution of generalized shunting inhibitory cellular neural networks with continuously distributed delays, ibid. 360 (2006), 122-130.

[11] - - - - Almost periodic solution of shunting inhibitory cellular neural networks with time varying and continuously distributed delays, ibid. 364 (2007), 17-28. 
[12] W. Lu and T. Chen, Global exponential stability of almost periodic solutions for a large class of delayed dynamical systems, Sci. China Ser. A Math. 8 (2005), 10151026.

[13] Q. Zhou, B. Xiao, Y. Yu and L. Peng, Existence and exponential stability of almost periodic solutions for shunting inhibitory cellular neural networks with continuously distributed delays, Chaos Solitons Fractals 34 (2007), 860-866.

Qiyuan Zhou, Changhong Zhao

Department of Mathematics

Hunan University of Arts and Science

Changde, Hunan 415000, China

E-mail: zhouqiyuan65@yahoo.com.cn

hongchangzhao@yahoo.com.cn

Received 28.7.2009

and in final form 8.4.2010 